$05,10,11$

\title{
Фрустрации и упорядочение в магнитных системах различной размерности
}

\author{
(C) Ф.А. Кассан-Оглы ${ }^{1}$, А.И. Прошкин ${ }^{1,2, \text { Ф }}$ \\ ${ }^{1}$ Институт фризики металлов УрО РАН им. М.Н. Михеева, \\ Екатеринбург, Россия \\ ${ }^{2}$ Уральский федееральный университет им. Б.Н. Ельцина, \\ Екатеринбург, Россия \\ ๑ E-mail: proshkin_ai@imp.uran.ru
}

На основании точных и численных расчетов в рамках моделей Изинга и Поттса на решетках различных типов и размерностей (одномерной, квадратной, треугольной, гексагональной, кагоме, простой кубической и объемноцентрированной) исследованы возникающие магнитные упорядочения, фазовые переходы и фрустрации при учете обменных взаимодействий между магнитными моментами на ближайших и вторых соседних узлах, а также внешнего магнитного поля. Установлены причины возникновения фрустраций и определены существенные особенности поведения фрустрированных систем, отличающие их от систем нефрустрированных.

Работа выполнена в рамках государственного задания ФАНО России (тема „Квант“ № 01201463332) при финансовой поддержке УрО РАН (проект № 15-8-2-10).

DOI: 10.21883/FTT.2018.06.45980.04M

\section{1. Введение}

За последние 15 лет к проблемам фрустраций в магнитных системах проявляется все возрастающий интерес, о чем свидетельствует бурный рост числа публикаций (см., например, [1]). Экспериментальный и теоретический материал по фрустрированным магнитным системам, как в низкоразмерных объектах, так и в реальных $3 D$-кристаллах и некристаллических веществах, весьма богат и изобилует новыми явлениями и необычными свойствами. Однако надлежащая интерпретация и теоретическое объяснение множества экспериментальных фактов и новых эффектов в настоящее время отсутствует, многие свойства фрустрированных систем еще недостаточно поняты. Численные расчеты разнообразными методами компьютерного моделирования фрустрированных систем часто приводят к ложным результатам из-за отсутствия надежных критериев истинности.

Главной задачей данного исследования являлось установление причин возникновения фрустраций в системе и нахождение конкретных значений физических параметров, определяющих упорядочена ли система или находится во фрустрированном состоянии. Такими параметрами служат знаки и величины обменных взаимодействий, а также направление и величина внешнего магнитного поля. Топология решетки также оказывает сильное влияние на упорядоченность либо фрустрированность системы при одних и тех же физических параметрах.

Другая важная задача исследования - установление существенных особенностей поведения фрустрированных систем, отличающих их от систем нефрустрированных.

В изинговской, 3-вершинной и стандартной 4-вершинной моделях Поттса исследовались магнитное упорядочение, фазовые переходы и фрустрации на $1 D$-, $2 D$ - и
$3 D$-решетках: цепочке, квадратной, треугольной, гексагональной, кагоме, простой кубической и объемноцентрированной кубической при учете обменных взаимодействий между магнитными моментами на ближайших и вторых соседних узлах, а также внешнего магнитного поля.

Для решения задач рассчитывались, в основном, энтропия и теплоемкость, используя аналитические формулы точных решений для максимального собственного значения трансфер-матрицы Крамерса-Ваннье. В отсутствие точных решений проводилось компьютерное моделирование в разных вариантах Монте-Карло и алгоритма Ванга-Ландау. С помощью расчетов энтропии, теплоемкости и намагниченности определялись точки фрустраций, фрустрационные поля и целые линии фрустраций.

Термин „фрустрация“ был привнесен в теорию магнетизма Тулузом в 1977 г. [2]. Фрустрации в физике твердого тела формулируются обычно как явление невозможности одновременной минимизации всех слагаемых гамильтониана в присутствие конкурирующих взаимодействий. Это приводит к сильному вырождению основного состояния системы (бесконечное число конфигураций, имеющих одинаковую наинизшую внутреннюю энергию) с ненулевой энтропией при нулевой температуре.

\section{2. Модели и основные формулы}

Все рассматриваемые модели на всех исследуемых решетках описываются гамильтонианом

$$
\mathscr{H}=-\frac{J}{2} \sum_{i, \Delta}\left(\mathbf{s}_{i} \cdot \mathbf{s}_{i+\Delta}\right)-\frac{J^{\prime}}{2} \sum_{i, \Delta^{\prime}}\left(\mathbf{s}_{i} \cdot \mathbf{s}_{i+\Delta^{\prime}}\right)-\sum_{i}\left(\mathbf{h} \cdot \mathbf{s}_{i}\right),
$$

где $J$ и $J^{\prime}$ - обменные взаимодействия между спинами на соседних и следующих узлах, $h-$ внешнее магнит- 


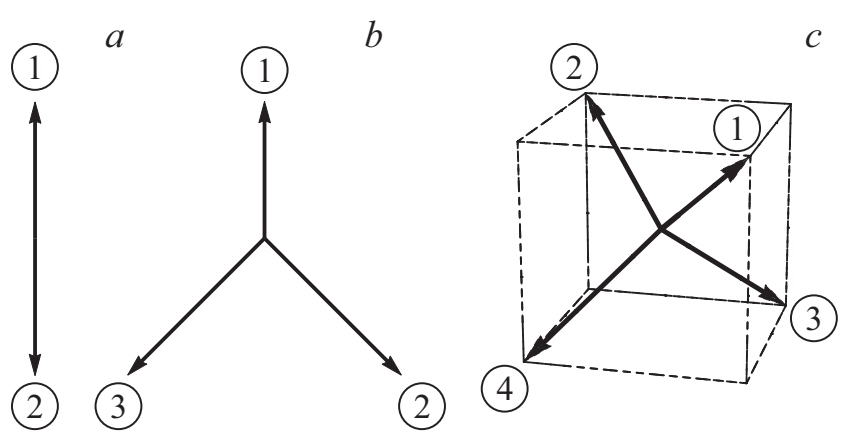

Рис. 1. $a-$ модель Изинга, $b-3$-х вершинная модель Поттса, $c-4-x$ вершинная стандартная модель Поттса.

ное поле, суммирование выполняется по всем $i$ узлам решетки, по ближайшим соседям $\Delta$ и вторым соседям $\Delta^{\prime}$ узла $i$. Векторы единичной длины $\mathbf{s}$ принимают два, три или четыре разрешенных значения соответственно в модели Изинга, 3- и 4-вершинной моделях Поттса (рис. 1). При наличии точных решений энтропия, теплоемкость и намагниченность выражаются только через максимальное собственное значение трансфер-матрицы Крамерса-Ваннье $\lambda$ с помощью формул

$$
S=\ln (\lambda)+\frac{T}{\lambda} \frac{\partial \lambda}{\partial T}, \quad C=T \frac{\partial S}{\partial T}, \quad M=\frac{T}{\lambda} \frac{\partial \lambda}{\partial h} .
$$

При отсутствии точных решений проводились численные расчеты модифицированными методами МонтеКарло, преимущественно в алгоритме Ванга-Ландау [3].

\section{3. Упорядочение и фрустрации на одномерной решетке}

На одномерной решетке в модели Изинга с учетом только взаимодействия между ближайшими соседями фрустраций не существует, имеет место фазовый переход при $T \rightarrow 0$ : в ферромагнитной модели - с сохранением периода трансляций; в антиферромагнитной - c удвоением периода трансляций. В антиферромагнитных моделях Поттса, наоборот, при учете только взаимодействия ближайших соседей фрустрации возникают, а фазовый переход при $T \rightarrow 0$ невозможен.

Учет взаимодействия между вторыми соседями порождает совершенно новые явления. В модели Изинга при учете двух типов конкурирующих взаимодействий между ближайшими и вторыми соседями: ферро-антиферро $\left(J>0, J^{\prime}<0\right)$ и антиферро-антиферро $\left(J<0, J^{\prime}<0\right)$ возникают фрустрации в точках $r=-0.5$ $\left(r=J^{\prime} / J\right)$ и $r=0.5$ соответственно, причем в обоих случаях энтропия при нулевой температуре равна логарифму золотого сечения $S_{T=0}=\ln [(1+\sqrt{5}) / 2] \sim 0.481$.

B 3-вершинной антиферромагнитной модели Поттса любое, даже сколь угодно малое ферромагнитное взаимодействие вторых соседей уничтожает фрустрации и порождает фазовый переход с удвоением периода трансляций; любое, даже сколь угодно малое, антиферромагнитное взаимодействие вторых соседей уничтожает фрустрации и порождает фазовый переход с утроением периода трансляций. При учете магнитного поля в 3-вершинной антиферромагнитной модели Поттса (направление поля совпадает с одним из направлений магнитных моментов) возникают фрустрации при $h_{f r}=-2 J$, в котором нультемпературная энтропия равна $\ln (2)$ [4].

В 4-вершинной стандартной антиферромагнитной модели Поттса любое, даже сколь угодно малое, положительное взаимодействие вторых соседей уничтожает фрустрации и порождает фазовый переход с удвоением периода трансляций. В отличие от 3-вершинной модели антиферромагнитное взаимодействие вторых соседей не может уничтожить фрустрации и породить фазовый переход. Основное состояние оказывается вырожденным с отличной от нуля энтропией при $T \rightarrow 0$, равной $\ln (2)$. Учет дополнительного антиферромагнитного взаимодействия между третьими соседями подавляет фрустрации [5].

В 3-вершинной ферромагнитной модели Поттса возникает полубесконечная линия фрустраций (фрустрационный параметр $\left.r=J^{\prime} / J\right)$, причем нультемпературная энтропия в точке фрустрации $(r=-0.5)$ равна $\ln (2)$, а при $r<-0.5: \ln (2) / 2$.

В 4-вершинной ферромагнитной стандартной модели Поттса также возникает полубесконечная линия фрустраций (фрустрационный параметр $\left.r=J^{\prime} / J\right)$, причем нультемпературная энтропия в точке фрустрации $(r=-0.5)$ равна $\ln [(1+\sqrt{13}) / 2]$, а при $r<-0.5$ : равна $\ln (3) / 2$.

Таким образом в 3- и 4-вершинных ферромагнитных моделях фрустраций не существует, а существует фазовый переход при $T \rightarrow 0$. Однако сильное взаимодействие вторых соседей $\left|J^{\prime} / J\right|>0.5$ порождает фрустрации и подавляет нультемпературный фазовый переход.

Мы исследовали также квантовую антиферромагнитную модель Изинга с произвольным значением спина и учетом внешнего магнитного поля на линейной решетке [6]. Получены общие аналитические выражения для фрустрационного поля, нультемпературных значений намагниченности и энтропии во фрустрационном поле, а также нультемпературной энтропии в точках фрустраций при учете конкурирующих взаимодействий между ближайшими и вторыми соседями.

Одномерная антиферромагнитная модель Изинга на цепочке спинов с учетом взаимодействий между ближайшими соседями в магнитном поле оказывается фрустрированной в магнитном поле $H_{f r}=2 s|J|$. При этом точные выражения для намагниченности и энтропии во фрустрирующем поле при $T \rightarrow 0$ как функции спина $s$ имеют вид

$$
\begin{gathered}
M_{f r}=\left(\frac{2 s-1}{4}\right)+\left(\frac{2 s+1}{4}\right) \frac{1}{\sqrt{1+8 s}}, \\
S_{f r}=\ln \left(\frac{1+\sqrt{1+8 s}}{2}\right) .
\end{gathered}
$$


В случае учета взаимодействий между ближайшими и вторыми соседями при отсутствии магнитного поля модель оказывается фрустрированной в точке $|r|=0.5$, где $r$ - отношение взаимодействия между вторыми соседями к таковому между ближайшими. В обоих случаях конкурирующих взаимодействий (антиферро-антиферро и ферро-антиферро) выражение для нультемпературной энтропии в точке фрустрации имеет вид

$$
\begin{aligned}
S_{f r}= & \ln \frac{1}{3}\left[1+\left(27 s-8+3 \sqrt{3} \sqrt{27 s^{3}-16 s}\right)^{1 / 3}\right. \\
& \left.+\left(27 s-8-3 \sqrt{3} \sqrt{27 s^{3}-16 s}\right)^{1 / 3}\right] .
\end{aligned}
$$

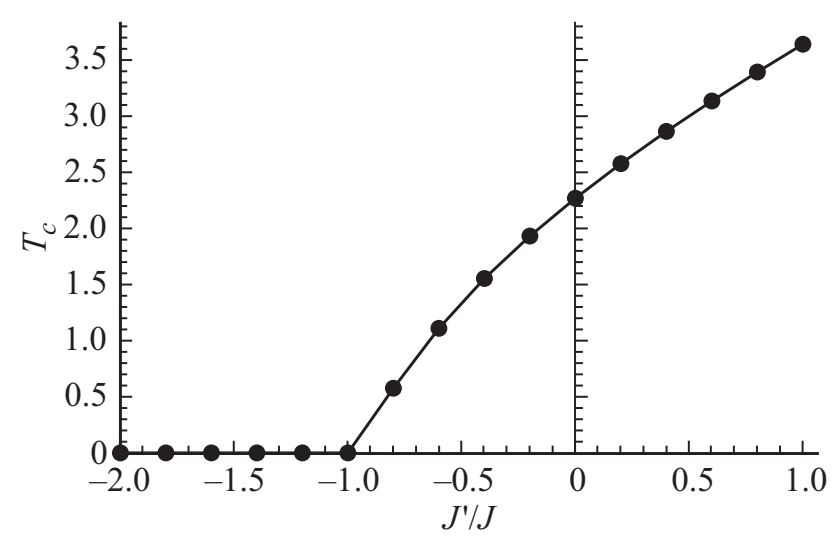

Рис. 3. Температура фазового перехода как функция $r=J_{2} / J_{1}$ в модели Изинга на треугольной решетке.

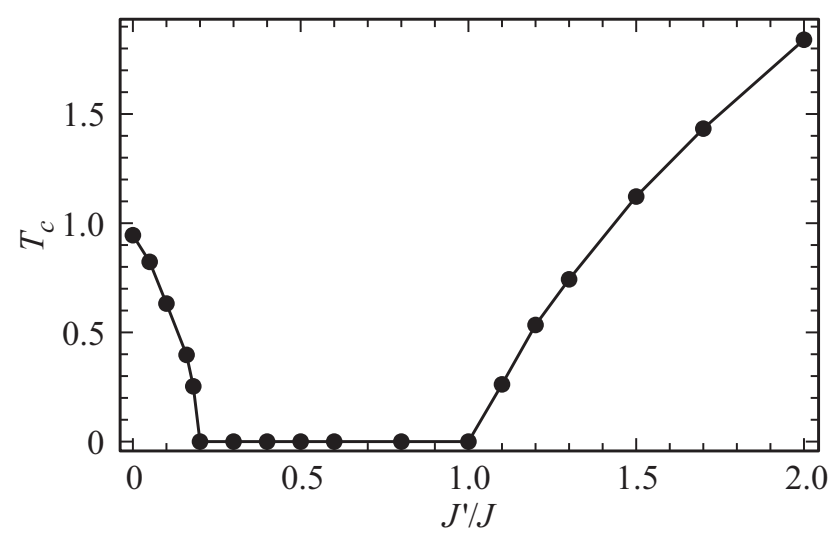

Рис. 4. Температура фазового перехода как функция $J^{\prime} / J$ в 3-вершинной модели Поттса на треугольной решетке [11].

распространен в литературе и получил не совсем удачное название квантового фазового перехода (QPT).

Второй тип фазовой диаграммы представлен на рис. 3 на примере модели Изинга на треугольной решетке и решетке кагоме. Фрустрационным параметром $r$, изменяющимся от $-\infty$ до $+\infty$, здесь также является отношение обменных взаимодействий между ближайшими соседями $J_{2} / J_{1}$ по двум разным направлениям. Однако существует одна особая фрустрационная точка $r=-1$, а также целый диапазон фрустраций на полубесконечной линии. Нультемпературная энтропия $S_{T \rightarrow 0}$ во фрустрационной точке не равна нулю (0.323 на треугольной решетке [8], 0.501 на решетке кагоме [9]). Во всем остальном диапазоне фрустрационного параметра $r \in(-1,+\infty)$ нультемпературная энтропия равна нулю.

Этот же тип фазовой диаграммы наблюдается в антиферромагнитной модели Изинга на квадратной решетке во внешнем поле при учете только взаимодействия между ближайшими соседями. Нультемпературная энтропия $S_{T=0} \sim 0.408$ во фрустрационном поле $h_{f r}=4$ [10].

На рис. 4 представлен третий, совершенно новый тип фазовой диаграммы, полученной в работе [11]. Расчеты проводились в 3-вершинной модели Поттса
Рис. 2. Температура фазового перехода как функция $J^{\prime} / J$ в модели Изинга на квадратной решетке. 


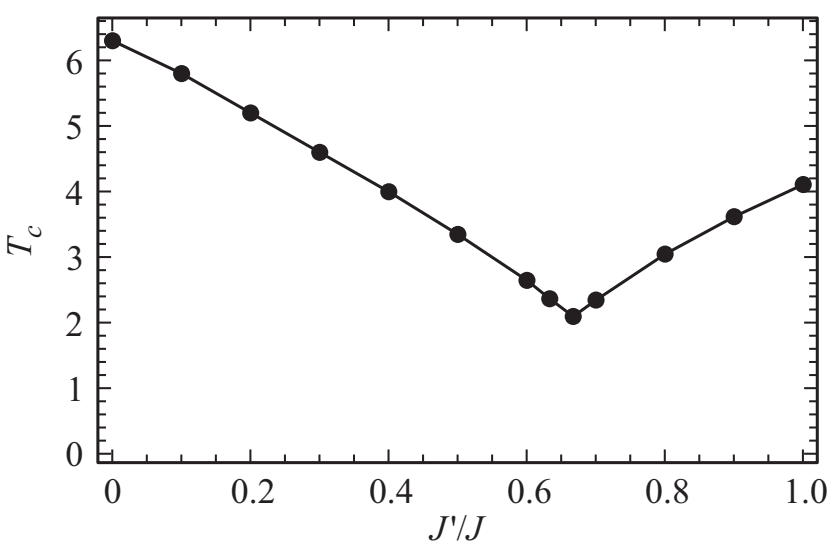

Рис. 5. Температура фазового перехода как функция $J^{\prime} / J$ в модели Изинга на ОЦК-решетке [12].

на треугольной решетке в двух вариантах конкурирующих обменных взаимодействий между ближайшими и вторыми соседями: 1) антиферромагнитное $J_{1}-$ антиферромагнитное $J_{2}$ и 2 ) ферромагнитное $J_{1}-$ антиферромагнитное $J_{2}$. В обоих случаях наблюдается не одна фрустрационная точка, а конечный диапазон фрустрационного параметра $r$ (от 0.2 до 1.0 в первом варианте и от -1.0 до -0.5 во втором), причем внутри того и другого диапазона нультемпературная энтропия $S_{T=0} \sim 0.301$.

Пример четвертого типа фазовой диаграммы представлен на рис. 5 на примере ОЦК-решетки в модели Изинга с конкурирующими взаимодействиями между ближайшими и вторыми соседями: антиферромагнитном $J_{1}$ и антиферромагнитном $J_{2}$ [12]. Фрустрирую- щая точка одна и равна $r=2 / 3$. Аналогичный вид имеет и диаграмма во втором варианте конкурирующих обменных взаимодействий: ферромагнитном $J_{1}$ и антиферромагнитном $J_{2}$ с точкой фрустрации, равной $r=-2 / 3$. Этот же тип фазовой диаграммы наблюдается на простой кубической решетке в обоих вариантах конкурирующих взаимодействий с точкой фрустрации $|r|=1 / 4$.

\section{5. Расщепление теплоемкости}

Мы обнаружили особое явление, присущее всем фрустрационным моделям на любых решетках: в непосредственной близости от точки фрустраций или фрустрационного поля теплоемкость расщепляется на острый лямбдаобразный онзагеровский пик и плавный куполообразный максимум. Острый пик, определяющий температуру фазового перехода, при приближении к точке фрустраций или фрустрационному полю стремится к нулю и исчезает точно в точке фрустраций или фрустрационном поле, а при удалении от точки фрустраций или фрустрационного поля, наоборот, исчезает куполообразный максимум.

Рис. 6, рассчитанный с помощью точного решения Онзагера [13], демонстрирует это явление. На рис. 6, a изображено поведение точки фазового перехода (сплошная линия) и положение куполообразного максимума (точки), как функция $r=J_{y} / J_{x}$. На рис. $6, b$ приведен расчет теплоемкости при $r=0.01$. Такое же расщепление теплоемкости обнаруживается при расчетах во всех фрустрированных моделях и решетках, в частности, на антиферромагнитной треугольной решетке и решетке кагоме.
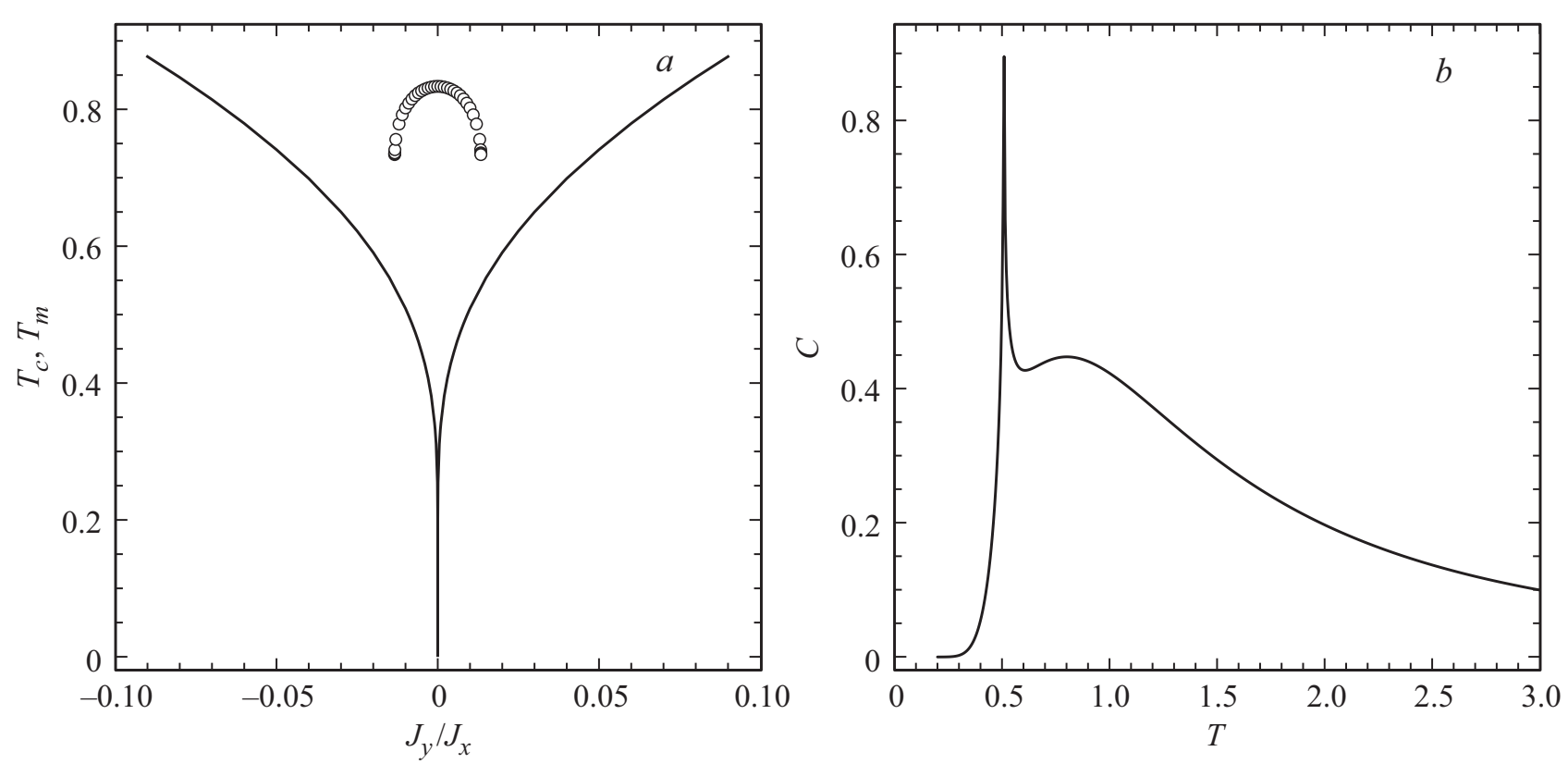

Рис. 6. Модель Изинга на квадратной решетке. $a$ - температура фазового перехода $T_{c}$ (сплошная линия) и положение пологого максимума $T_{m}$ (точки). $b$ - теплоемкость при $J_{y} / J_{x}=0.01$. 

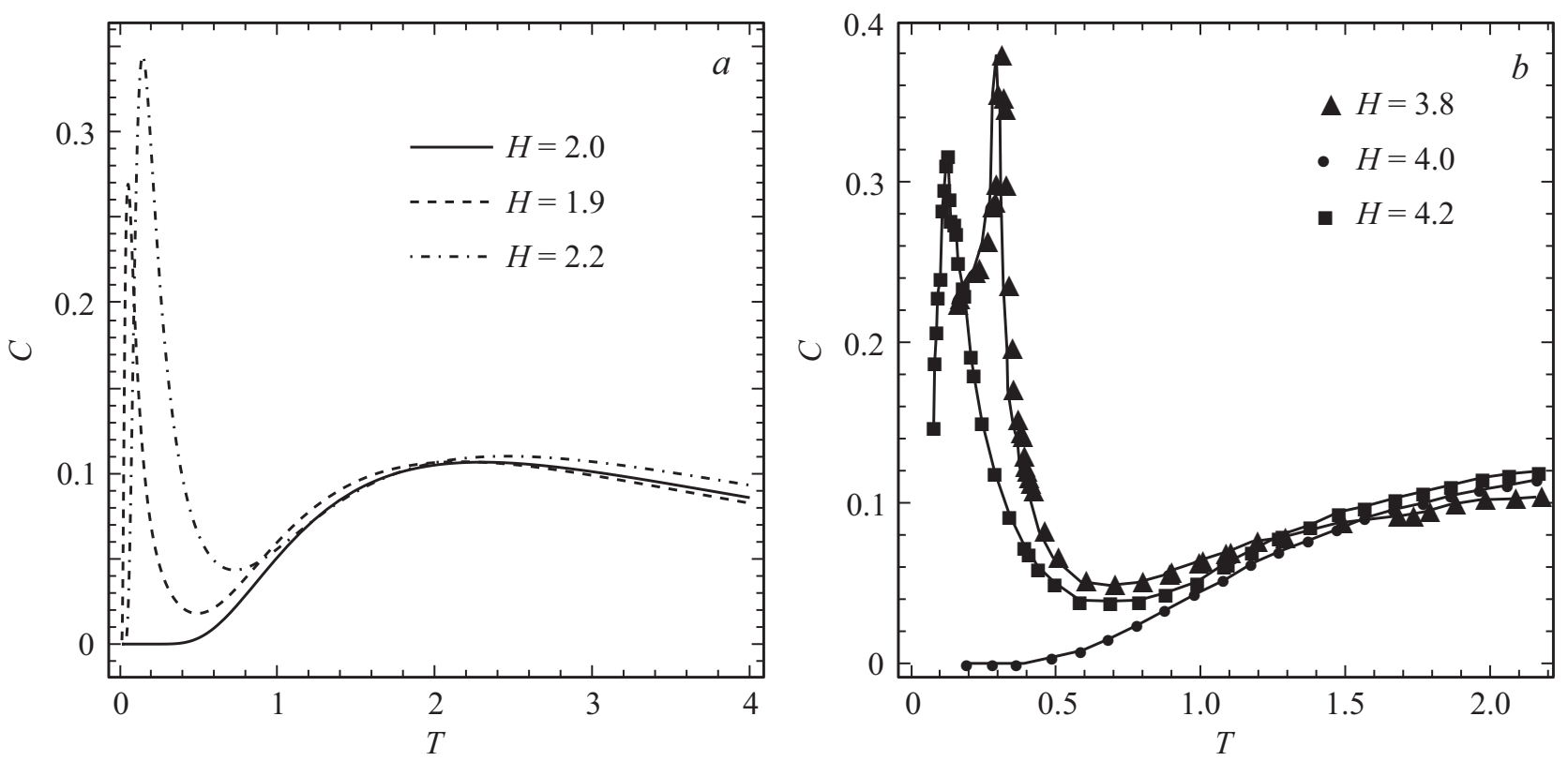

Рис. 7. Теплоемкость в модели Изинга на линейной $(a)$ и квадратной решетках $(b)$.

Рис. 7 демонстрирует, в качестве примера, расщепление теплоемкости на линейной цепочке и квадратной решетке в антиферромагнитной модели Изинга вблизи фрустрационного поля.

Такое расщепление магнитного вклада в теплоемкость и сдвиги острого пика и куполообразного максимума наблюдаются и в реальных $3 D$-кристаллах, в которых магнитные атомы занимают ГЦК-решетку, например, в монохалькогениде TmTe [14] и в монопниктиде $\mathrm{ErBi}$ [15]. Авторы обеих работ отмечают, что им не удалось описать расщепление теплоемкости теоретически.

\section{6. Частичное упорядочение}

Во фрустрированных системах наблюдается еще одно явление, тесно связанное с общими свойствами энтропии - это частичное упорядочение, или упорядочение с понижением размерности. В рассматриваемых моделях нультемпературная или остаточная энтропия (при $T \rightarrow 0)$, приходящаяся на один узел решетки, равна $\ln (n) / N$, где $N$ - число узлов решетки, а $n-$ число конфигураций с наинизшей внутренней энергией, то есть таких, которые выживают при $T \rightarrow 0$. Если $n$ конечно, то в термодинамическом пределе $(N \rightarrow 0)$ энтропия равна нулю, а в системе наблюдается упорядочение. Если же $n$ бесконечно и порядка $q^{N}(q-$ число разрешенных состояний на узле) то нультемпературная энтропия не равна нулю, и упорядочения нет даже при $T \rightarrow 0$, что соответствует фрустрациям.

На рис. 8, демонстрирующем пример частичного упорядочения на треугольной решетке в модели Изинга с антиферромагнитными взаимодействиями по трем направлениям (причем $J_{3}<J_{1}=J_{2}$ ), представлена одна из

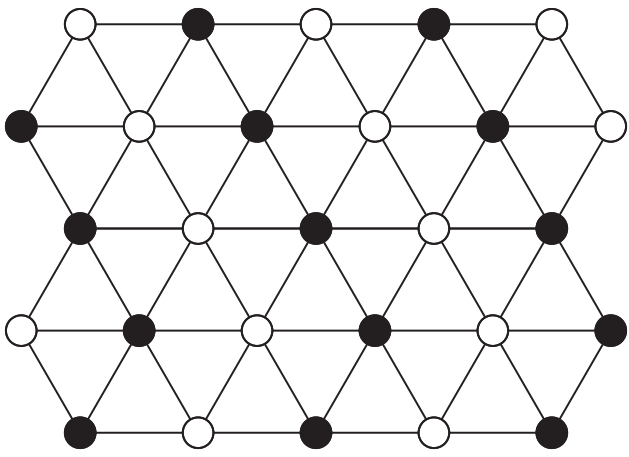

Рис. 8. Частичное упорядочение на треугольной решетке.

бесконечного множества конфигураций, имеющих наинизшую энергию при $T=0$. Видно, что конфигурация представляет структуру, составленную из цепочек, упорядоченных антиферромагнитно в горизонтальном направлении, и неупорядоченных по другим направлениям.

Рис. 9 демонстрирует одновременно два примера частичного упорядочения на квадратной решетке в модели Изинга. Первый соответствует учету взаимодействия только между ближайшими соседями, причем $J_{y}>0$, $J_{x}=0$. Второй пример соответствует учету взаимодействий между ближайшими и вторыми соседями, причем оба взаимодействия $J_{1}$ и $J_{2}$ антиферромагнитные, а фрустрирующий параметр $r=J_{2} / J_{1}=0.5$. Нультемпературная энтропия, приходящаяся на один узел решетки, в первом случае равна $\ln (2) / N_{y}$ и соответственно равна нулю. Во втором случае, нультемпературная энтропия другая. Она равна $\ln [(1+\sqrt{5}) / 2] / N_{x}$ и в термодинамическом пределе также стремится к нулю. Совершенно 


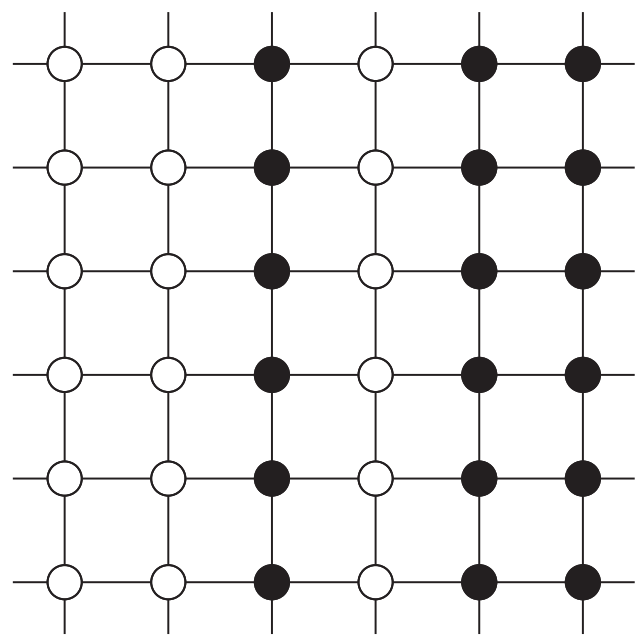

Рис. 9. Частичное упорядочение на квадратной решетке.

очевидно, что на всех $2 D$-решетках в любых моделях частичное упорядочение сопровождается одним типом понижения размерности, а именно, с двумерного на одномерное. В трехмерном случае возможны три типа понижения размерности. Первый, с $3 D$ на $2 D$, который в точке фрустраций должен сопровождаться ненулевым значением температуры перехода. Второй, с $3 D$ на $1 D$ с нулевым значением температуры перехода в точке фрустраций. Третий, двухстадийное понижение размерности, сначала с $3 D$ на $2 D$ с ненулевым значением температуры перехода, а затем с $2 D$ на $1 D$ с нулевым значением температуры перехода. Поэтому определение и поиски частичного упорядочения на $3 D$-решетках представляет собой довольно сложную задачу.

\section{7. Конкурирующие и неконкурирующие взаимодействия}

В обширной литературе по компьютерному моделированию обычно считается, что конкурирующие взаимодействия между ближайшими и вторыми соседями могут быть в двух вариантах: ферро-антиферро $(J>0$ и $\left.J^{\prime}<0\right)$, либо антиферро-антиферро $\left(J<0\right.$ и $\left.J^{\prime}<0\right)$. Это справедливо для модели Изинга на решетках всех размерностей, для 3-вершинной модели Поттса на треугольной решетке, для 4-вершинной стандартной модели Поттса на ГЦК-решетке и многих других. Однако это не всегда так. Совокупное влияние топологии решетки и применяемой модели может приводить к противоположному результату.

Рассмотрим, например, 3-вершинную модель Поттса с антиферромагнитными обменными взаимодействия между ближайшими $J$ и вторыми соседями $J^{\prime}$ на треугольной решетке и решетке кагоме. В этом случае оба взаимодействия (в отличие от модели Изинга) требуют не антипараллельного расположения спинов в любой паре, а расположения спинов под углами 120 , либо $240^{\circ}$. Будем считать, что взаимодействие между ближайшими соседями много больше, чем между вторыми соседями. Тогда мы обязаны расположить спины на всех ближайших соседях под углами 120, либо $240^{\circ}$. На рис. 10 (треугольная) и рис. 11 (кагоме) это требование выполнено на обеих решетках. Однако на треугольной решетке все пары вторых соседей расположены параллельно, что противоречит требованию обменного взаимодействия $J^{\prime}$, т.е. возникла конкуренция. В противоположность этому, на решетке кагоме все пары вторых соседей уже расположены под углами $120^{\circ}$ либо $240^{\circ}$, что отвечает требованию обменного взаимодействия $J^{\prime}$. Таким образом, на решетке кагоме антиферромагнитные взаимодействия между ближайшими и вторыми соседями являются неконкурирующими.

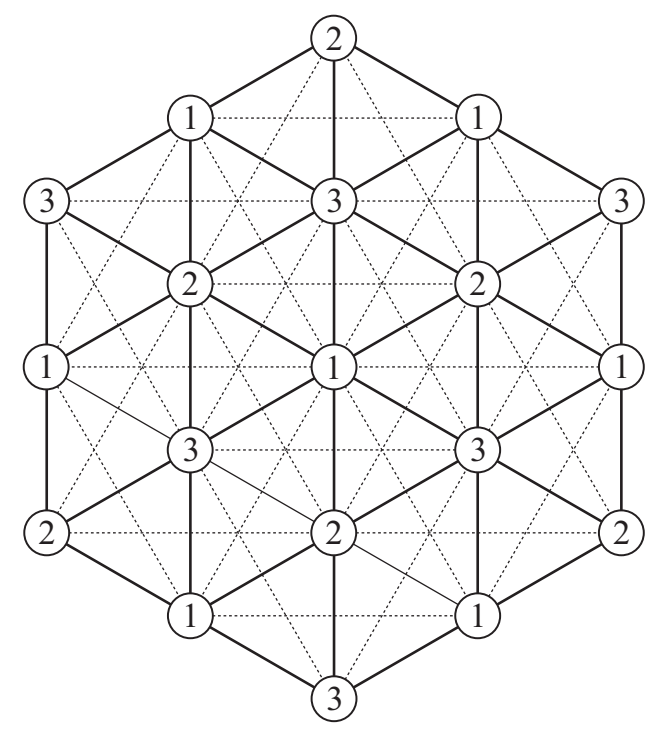

Рис. 10. 3-вершинная модель Поттса на треугольной решетке.

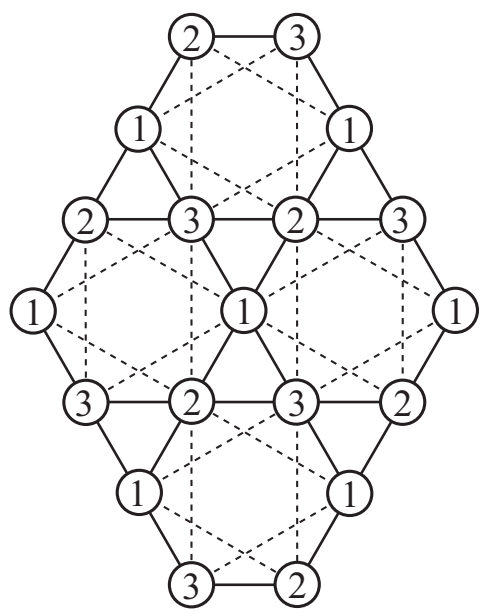

Рис. 11. 3-вершинная модель Поттса на решетке кагоме. 


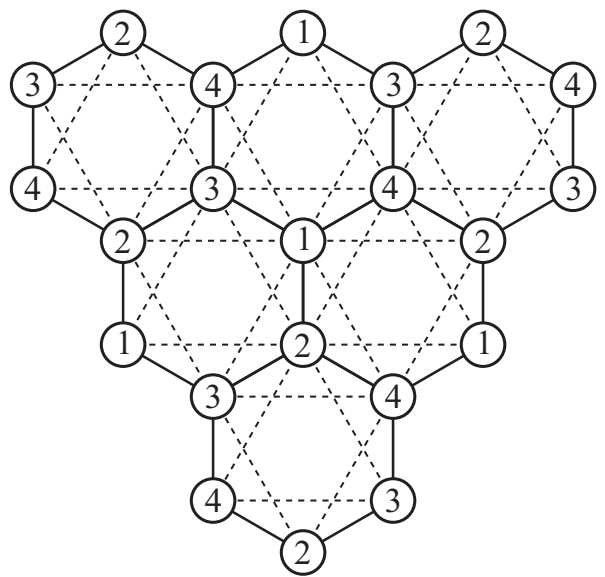

Рис. 12. 4-вершинная стандартная модель Поттса на гексагональной решетке.

На рис. 12 приведен еще один пример неконкурирующих антиферромагнитных взаимодействий $J$ и $J^{\prime}$ на гексагональной решетке в 4-вершинной стандартной модели Поттса. В данном случае оба взаимодействия (в отличие от модели Изинга) требуют не антипараллельного расположения спинов в любой паре, а расположения спинов под углом $\arccos (-1 / 3)$, и это требование уже выполнено для любых численных значений $J$ и $J^{\prime}$. Аналогичное явление наблюдается в 4-вершинной стандартной модели Поттса на треугольной решетке.

\section{8. Заключение}

В данной работе в рассмотренных моделях на разных решетках исследовано возникновение самых разнообразных вариантов фазовых переходов и фрустраций от отдельных точек фрустраций и фрустрационных полей до конечных и бесконечных линий фрустраций в пространстве параметров обменных взаимодействий и внешнего поля. В некоторых случаях фрустрации полностью подавляют фазовый переход для любых численных значений обменных взаимодействий. Подробно обсуждены уникальные особенности, присущие фрустрированным системам, которые не наблюдаются в системах без фрустраций. Это и разные типы фазовых диаграмм, и частичное упорядочение (упорядочение с понижением размерности). Наиболее поразительное свойство систем с фрустрациями - расщепление теплоемкости вблизи точек фрустраций и фрустрационных полей на острый лямбдаобразный онзагеровский пик и плавный куполообразный максимум, наблюдаемое во всех моделях на всех решетках, а также в различных экспериментах на реальных $3 D$-кристаллах. Это и тот факт, что одни и те же обменные взаимодействия могут быть как конкурирующими, так и неконкурирующими, приводит к возникновению, либо к подавлению фрустраций.
Следует отметить еще одну важную особенность фрустрированных систем - особые трудности численных расчетов при компьютерном моделировании, главной причиной которых является наличие множества конфигураций с весьма близкими значениями внутренней энергии именно вблизи точек фрустраций и фрустрационных полей. Вследствие этого, а также отсутствия надежных критериев истинности, многие подходы и численные методы расчета, отлично работающие при описании систем без фрустраций, часто приводят к явным ошибкам и ложным эффектам при попытках описать поведение фрустрированных систем. Так, например, в работе Юу [16], посвященной исследованию антиферромагнитной модели Изинга на одиннадцати двумерных архимедовых решетках с учетом только взаимодействия между ближайшими соседями, и опубликованной в престижном журнале Physical Reviev E, приведены численные значения нультемпературной энтропии для всех одиннадцати решеток, в том числе и для квадратной и гексагональной, причем для этих решеток нультемпературная энтропия равна $\ln (2)$, что является ошибкой, поскольку из точных решений Онзагера [13] и Гутаппеля [8] следует, что она равна нулю в том и другом случае. В этом отношении весьма примечательна работа авторитетного специалиста в области компьютерного моделирования Биндера [17], посвященная исследованию ГЦК-решетки также с учетом взаимодействия только между ближайшими соседями. В этой работе Биндер сделал следующие замечания:

1. Фазовая диаграмма отлична от всех предыдущих расчетов.

2. Все предыдущие подходы не согласуются с полученной диаграммой.

3. В заключение я нахожу серьезные недостатки в предыдущих исследованиях упорядочения на ГЦКрешетке, таких как методом кластерного варьирования,

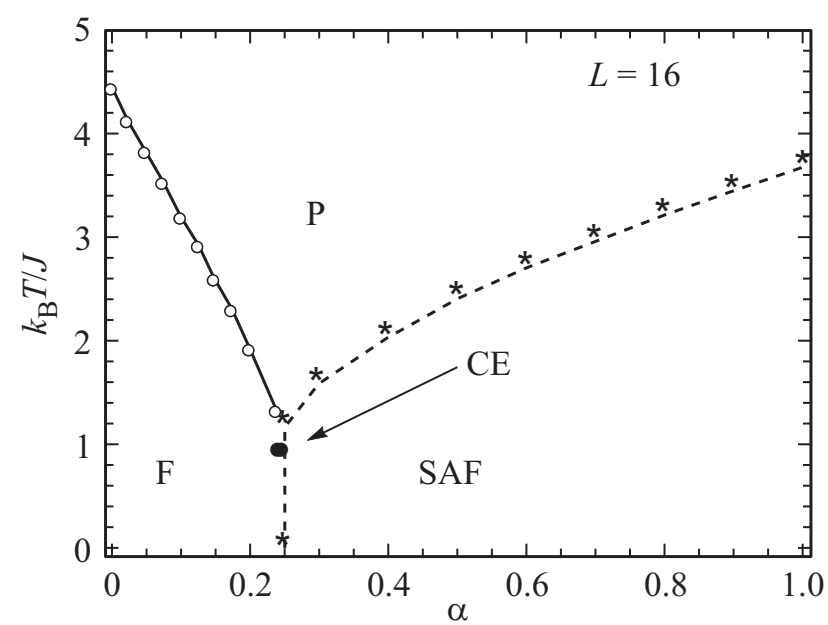

Рис. 13. Температура фазового перехода как функция фрустрационного параметра $\alpha=J_{2} / J_{1}$ в модели Изинга на простой кубической решетке [18]. 

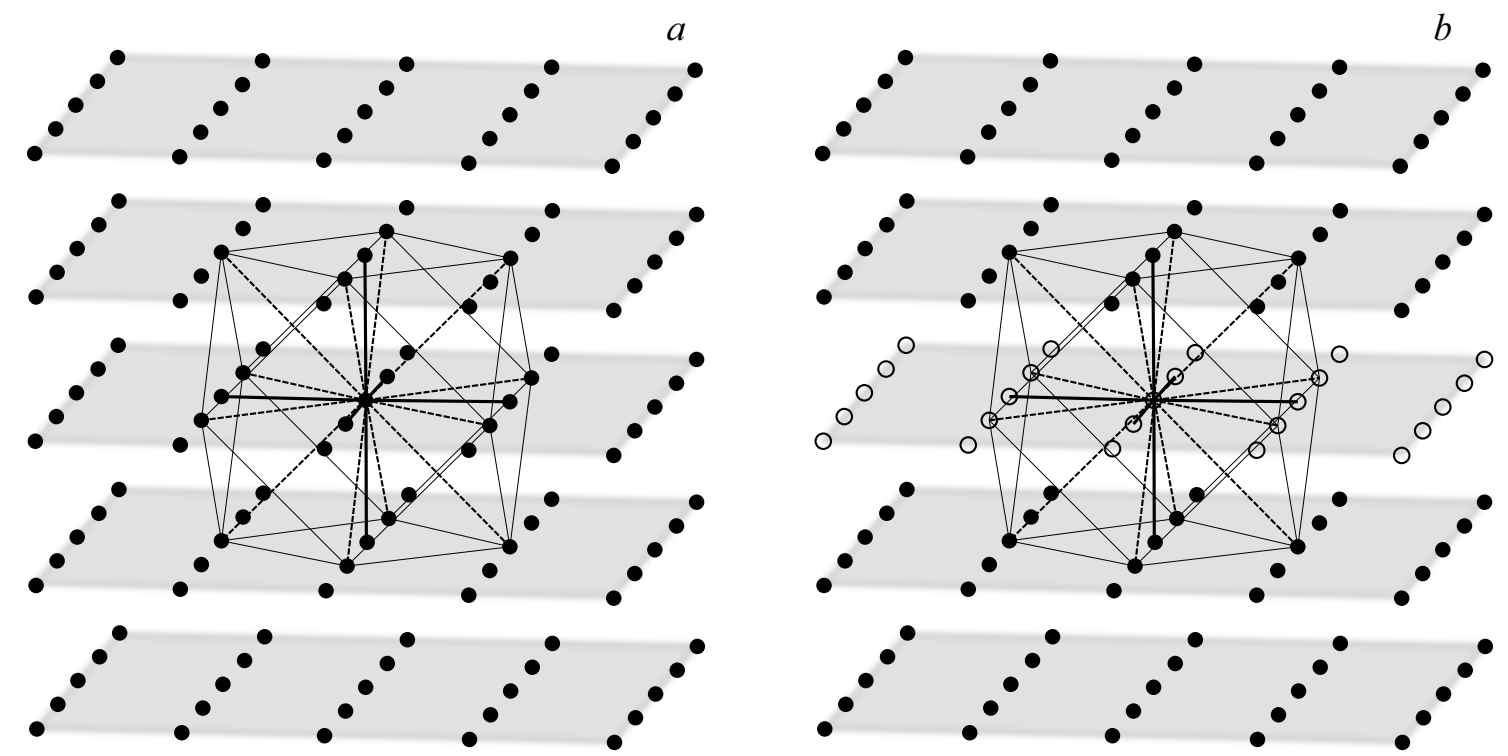

Рис. 14. Частичное упорядочение в модели Изинга на простой кубической решетке с конкурирующими ферромагнитными $J$ и антиферромагнитными $J^{\prime}$ взаимодействиями.

которые не учитывают должным образом „фрустрационные эффекты“.

Таким образом, следует учитывать все существенные особенности, присущие фрустрированным системам. В качестве примера рассмотрим проблему исследования на простой кубической решетке при учете ферромагнитного взаимодействия между ближайшими соседями $J_{1}$ и антиферромагнитного взаимодействия между вторыми соседями $J_{2}$ в модели Изинга, дискутируемую между авторами работ [18] и [19]. На рис. 13 приведена фазовая диаграмма из работы [18], на которой присутствуют два значения для температуры перехода в точке фрустраций со значением фрустрационного параметра $\alpha=J_{2} / J_{1}=0.25$. Главное различие результатов работ [18] и [19] заключается только в нулевом и ненулевом значении этой температуры перехода. При учете эффекта частичного упорядочения можно сделать вывод, что в первом случае должно наблюдаться понижение размерности из $3 D$ в $1 D$, а во втором из $3 D$ в $2 D$. Анализ внутренней энергии, свидетельствует о том, что единственно возможный вариант в простой кубической решетке, а именно, с $3 D$ на $2 D$, происходит с упорядочением трех семейств плоскостей типа $\{100\}$. Следовательно, температура перехода в точке фрустраций должна быть ненулевой. Рис. 14 иллюстрирует результаты этого анализа.

\section{Список литературы}

[1] H.T. Diep. Frustrated spin systems. World Scientific (2013). $644 \mathrm{c}$.

[2] G. Toulouse. Commun. Phys. 2, 115 (1977).

[3] F. Wang, D.P. Landau. Phys. Rev. E 64, 056101 (2001).

[4] F.A. Kassan-Ogly. Phase Transitions 71, 39-55 (2000).
[5] A.I. Proshkin. JMMM 383, 13-18 (2015).

[6] A. Proshkin, F. Kassan-Ogly. Mater. Sci. Forum 845, 93 (2016).

[7] A. Kalz, A. Honecker, S. Fuchs, T. Pruschke. Eur. Phys. J. B 65, 533 (2008).

[8] R.M.F. Houtappel. Physica 16, 425 (1950).

[9] K. Kanô. Prog. Theor. Phys. 10, 158 (1953).

[10] F.A. Kassan-Ogly, A.K. Murtazaev, A.K. Zhuravlev, M.K. Ramazanov, A.I. Proshkin. JMMM 384, 247-254 (2015).

[11] А.Б. Бабаев, М.А. Магомедов, А.К. Муртазаев, Ф.А. Кассан-Оглы, А.И. Прошкин. ЖЭТФ 149, 357 (2016).

[12] А.К. Муртазаев, М.К. Рамазанов, Ф.А. Кассан-Оглы, Д.Р. Курбанова. ЖЭТФ 147, 127 (2015).

[13] L. Onsager. Phys. Rev. 65, 117 (1944).

[14] T. Matsumara, H. Shida, T. Suzuki. Physica B: 230-232, 738 (1997).

[15] H. Wada, H. Imai, M. Shiga. J. Alloys Compd. 218, 73 (1995).

[16] U. Yu. Phys. Rev. E 91, 062121 (2015).

[17] K. Binder. Phys. Rev. Lett. 45, 811 (1980).

[18] O.D.R. Salmon. Int. J. Mod. Phys. B 27, 1350162 (2013).

[19] R.A. dos Anjos. Phys. Rev. E 76, 022103 (2007). 